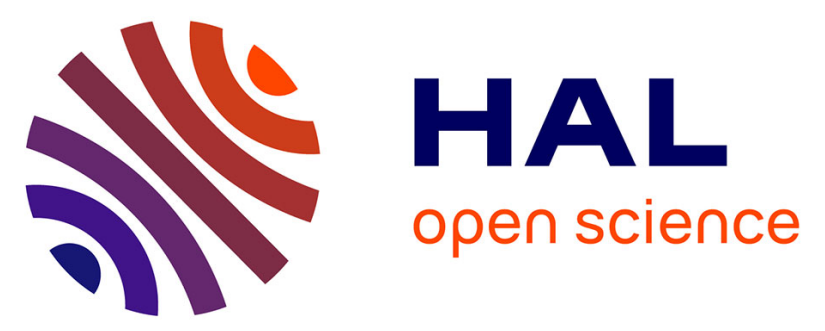

\title{
X-ray Structure of the Human Karyopherin RanBP5, an Essential Factor for Influenza Polymerase Nuclear Trafficking
}

Christopher Swale, Bruno da Costa, Laura Sedano, Frederic Garzoni, Andrew Mccarthy, Imre Berger, Christoph Bieniossek, Rob W H Ruigrok, Bernard Delmas, Thibaut Crépin

\section{To cite this version:}

Christopher Swale, Bruno da Costa, Laura Sedano, Frederic Garzoni, Andrew Mccarthy, et al.. X-ray Structure of the Human Karyopherin RanBP5, an Essential Factor for Influenza Polymerase Nuclear Trafficking. Journal of Molecular Biology, 2020, 432 (10), pp.3353-3359. 10.1016/j.jmb.2020.03.021 . hal-02555361

\section{HAL Id: hal-02555361 https://hal.univ-grenoble-alpes.fr/hal-02555361}

Submitted on 23 Nov 2020

HAL is a multi-disciplinary open access archive for the deposit and dissemination of scientific research documents, whether they are published or not. The documents may come from teaching and research institutions in France or abroad, or from public or private research centers.
L'archive ouverte pluridisciplinaire HAL, est destinée au dépôt et à la diffusion de documents scientifiques de niveau recherche, publiés ou non, émanant des établissements d'enseignement et de recherche français ou étrangers, des laboratoires publics ou privés.

$$
\text { Copyright }
$$




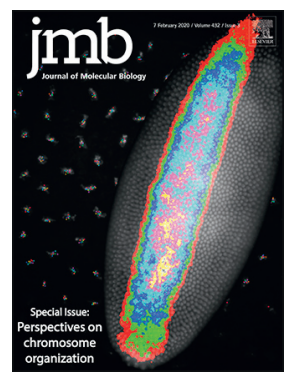

\title{
X-ray Structure of the Human Karyopherin RanBP5, an Essential Factor for Influenza Polymerase Nuclear Trafficking
}

\section{Christopher Swale ${ }^{1,2, \dagger}$, Bruno Da Costa ${ }^{3}$, Laura Sedano ${ }^{3}$, Frédéric Garzoni ${ }^{2, \ddagger}$, Andrew A. McCarthy ${ }^{2}$, Imre Berger ${ }^{2,4}$, Christoph Bieniossek ${ }^{5}$, Rob W.H. Ruigrok ${ }^{1}$, Bernard Delmas ${ }^{3}$ and Thibaut Crépin ${ }^{1}$}

1 - Institut de Biologie Structurale (IBS), University Grenoble Alpes, CEA, CNRS, 38044 Grenoble, France

2 - EMBL Grenoble Outstation, 71 Avenue des Martyrs, BP181, F-38042 Grenoble Cedex 9, France

3 - Virologie et Immunologie Moléculaires, INRA, Université Paris-Saclay, 78350 Jouy-en-Josas, France

4 - Max Planck Centre for Minimal Biology, University of Bristol, Clifton BS8 1TD, United Kingdom

5 - Roche Innovation Centre, Basel, Switzerland F. Hoffmann-La Roche AG, Grenzacherstrasse 124, CH-4070 Basel, Switzerland

Correspondence to Thibaut Crépin: Institut de Biologie Structurale (IBS, UMR5075 CEA-CNRS-UGA), 71 avenue des Martyrs, CS 20192, 38042 Grenoble, France. thibaut.crepin@ibs.fr

https://doi.org/10.1016/j.jmb.2020.03.021

Edited by Dr. Edward Lemke

\begin{abstract}
Here, we describe the crystal structures of two distinct isoforms of ligand-free human karyopherin RanBP5 and investigate its global propensity to interact with influenza A virus polymerase. Our results confirm the general architecture and mechanism of the IMB3 karyopherin- $\beta$ subfamily whilst also highlighting differences with the yeast orthologue Kap121p. Moreover, our results provide insight into the structural flexibility of $\beta$-importins in the unbound state. Based on docking of a nuclear localisation sequence, point mutations were designed, which suppress influenza PA-PB1 subcomplex binding to RanBP5 in a binary protein complementation assay.
\end{abstract}

(C) 2020 Elsevier Ltd. All rights reserved.

In eukaryotic cells, the compartmentalization of the nucleus and cytoplasm by the nuclear membrane has enabled spatial separation of cellular processes. The genome is maintained, replicated and transcribed within the nucleus, whilst proteins are synthetized and processed in the cytoplasm. Both compartments, however, rely on transmembrane traffic as many factors are synthetized in one compartment but then function within the other, including tRNAs, ribosomes, nuclear proteins and other elements. The transport between both compartments is controlled by the nuclear pore complex (NPC), a supramolecular assembly of nucleoporin proteins anchored within the nuclear membrane, with an approximate mass of $125 \mathrm{MDa}$ in vertebrates [1,2]. The NPC acts as a molecular sieve, which only permits the passive diffusion of small molecules with a molecular mass below $40 \mathrm{kDa}$, although in practice, most cargo (proteins, RNA, ribonucleocomplexes) are transported actively through the NPC [3]. This active transport is controlled by transport proteins called karyopherins, which import and export cargo to and from the nucleus [4,5]. Amongst importins, two classes have been defined, the importin- $\beta$ class and their adaptor, the importin- $\alpha$ class. Importin recruitment onto cargo proteins is enabled by specific peptide motifs called nuclear localisation signals (NLS), belonging to two separate classes [6], classical NLS signals and nonclassical or PY NLS signals. Additionally to the interaction with the NLS motif, recent karyopherins$\beta /$ cargo-protein structures, notably Imp13/Mago-Y14 [7] and Imp9/H2A-H2B [8], have demonstrated that these complexes are dependent on multiple interactions, mediated through complex 3D interfaces, which involve residues well beyond the NLS peptide stretch.

The import process is controlled by Ran, which is found mostly within the nucleus, bound to GTP. RanGTP acts as an import complex release switch by 
directly interacting with $\beta$-karyopherins upon their entry within the nucleus [9-11]. After releasing the cargo, $\beta$-karyopherins bound to Ran-GTP are shuttled back to the cytoplasm where the interaction with Ran-GAP activates the hydrolysis of GTP into GDP, actively triggering a dissociation of Ran from its bound importin. The entire process enables for a complete recycling of the components.

When influenza infection occurs, the replicative ribonucleoproteins, a complex between vRNA, nucleoprotein and the RNA-dependant RNA polymerase (RdRp) will migrate to the nucleus of the infected cell to perform both transcription and replication $[12,13]$. De novo ribonucleoprotein assembly also takes place in the nucleus [14] requiring the import of all protein components following translation. The RdRp trimer [15-19], composed of PA, PB1 and PB2, has been shown to assemble in two distinct modules $[20,21]$. The PA-PB1 sub- complex is imported by the karyopherin RanBP5 $[22,23]$, whilst PB2 is imported by an importin- $\alpha$ mediated pathway [24].

Baculovirus-infected insect cells were used to express recombinant human RanBP5, the mammalian homologue of fungal Kap121, as previously described [23]. RanBP5 was first crystallised in a $P 22_{1} 2_{1}$ space group using the full-length construct (isoform-3, residues 1 to 1116). A first model was obtained by molecular replacement at $3.0 \AA$ resolution, using the yeast Kap121p structure $(29 \%$ sequence identity) $[25,26]$ as a starting model. Kap121p was separated in three parts, using the multiple domain search mode of Phaser [27] (Supplementary Data). This first crystal form provided a preliminary model and included an $\mathrm{N}$-terminal stretch of 21 amino acid residues, which was presumably unstructured and could not be traced in the electron density. From this first model, we then (a)

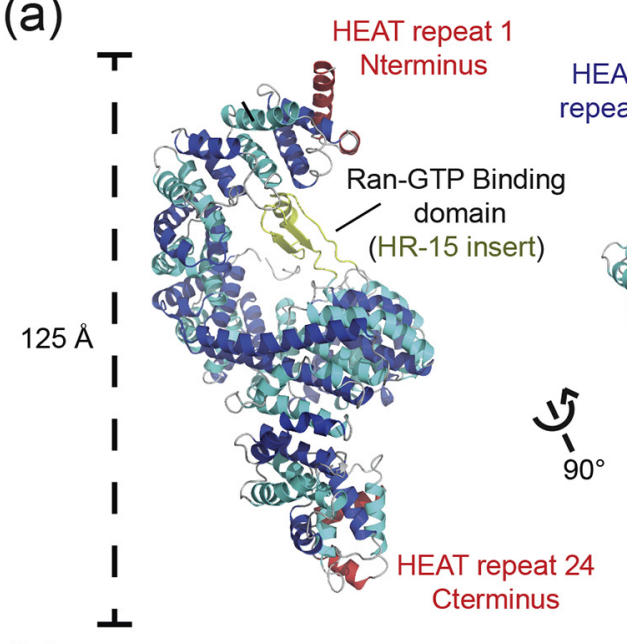

(b)

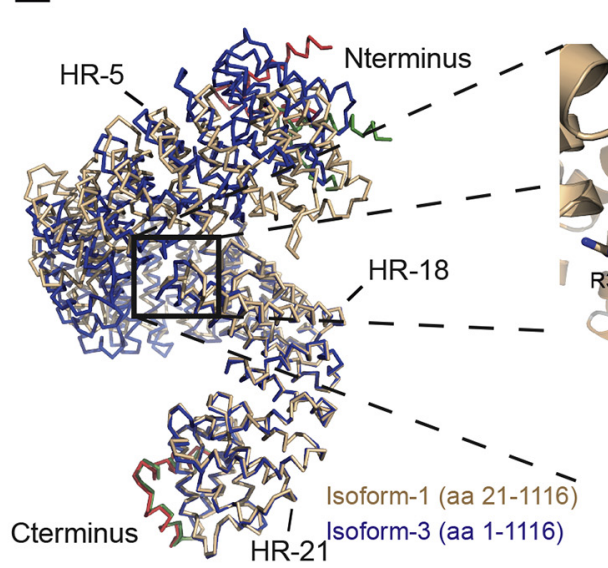

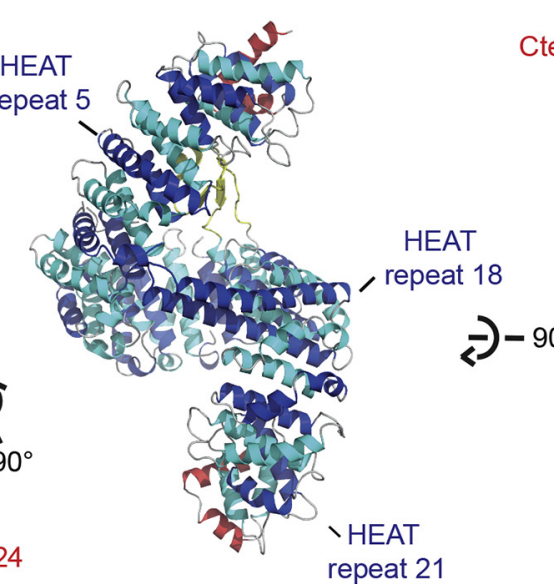

Cterminus

$-90^{\circ}$
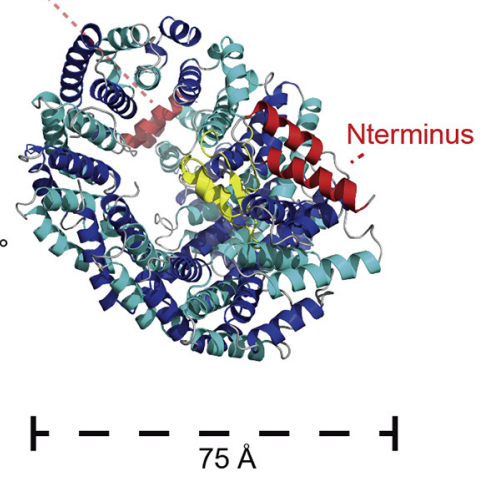

repeat 21

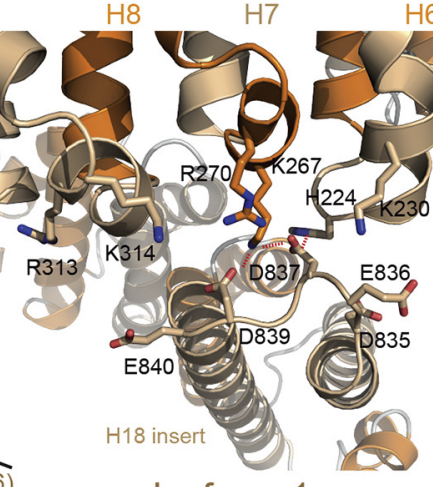

Isoform-1

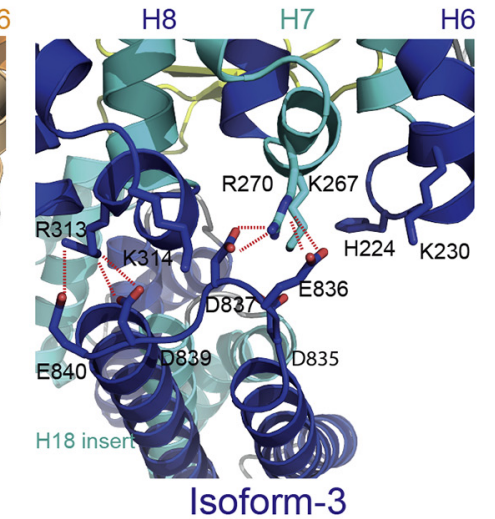

Figure 1. Structure of RanBP5 in the unbound conformation. (a) Architecture of the full-length RanBP5 (isoform-1) is depicted in a cartoon representation. N-terminal and C-terminal HEAT repeats (HR-1 and HR-24) are coloured in red, HR2 to -23 are coloured alternately in cyan and blue, and the HR-15 insert, or Ran GTP binding domain, is coloured in dark yellow. (b) Crystal structures of isoform-1 (orange) and isoform-3 (blue) are superimposed for comparison, aligned on the C-terminal region (residues 800 to 1116). A zoom-in of the HR-18 extension or "clamp" interacting by forming salt bridges with the N-terminal part of RanBP5 is shown for both conformations. 
designed a second construct based on the isoform-1 of RanBP5 (corresponding to residues 21 to 1116 of the isoform-3) lacking the unstructured region. We produced and crystallised this construct in again a $P 2{ }_{1} 2_{1} 2_{1}$ space group but different cell parameters, with a maximum resolution of $2.5 \AA$. We used the two datasets to implement gradually the entire model. Isoform- 3 and -1 data collection and structure resolution statistics are presented in the Table 1. Overall, RanBP5 adopts a right-handed solenoid form (Figure 1(a)) comparable with $\beta$-importins previously crystallised [26,28-31]. Like the yeast karyopherin Kap121p (comparison shown in Figure S1), RanBP5 is organised in 24 tandem HEAT repeats (HR). HEAT repeats include two antiparallel a-helices composed of 8 to 21 amino acid residues connected by loops of 3 to 6 residues. Systematically, one of the antiparallel $\alpha$-helices is exposed to the outer part of the solenoid, whilst the other is exposed to the inner region. N-terminal HR-1 and C-terminal HR-24 cap the ends of the solenoid by stacking at virtually $90^{\circ}$ against the adjacent HEAT repeats. RanBP5 displays a series of extensions within or in between HR-8, HR15, and HR-18, similar to Kap121p. The HR-8 acidic loop (residues 332 to 352 ) appears to be flexible in our models, with defined electron density only observed for a short part of the sequence, which stacks at the interface between HR-10 and HR-11 in the isoform-3. RanBP5 also possesses an insertion module within HR-15, which can be described as a globular domain composed of two antiparallel $\beta$-strands and one short $\alpha$-helix. The HR-15 insert is not visible in the isoform-1 structure. This insertion module is known to directly interact with Ran [26]. Together with the N-terminal HR-1, HR-2, HR-3 and HR-6, HR-7 and HR-8, this insertion module helps discriminate between RanGTP and Ran-GDP, thereby acting as a regulator of Ran-GTP binding. The last insertion module is in HR-18 and comprised two long a-helices of 29 amino acid residues each (Figure S1). This feature extends towards and interacts with the $\mathrm{N}$-terminal domain of RanBP5 through an acidic loop region (835-DEDYDE-840), which we define as the

Table 1. Crystallographic data collection and refinement statistics

\begin{tabular}{|c|c|c|}
\hline & RanBP5 isoform-3 (residues 1 to 1116 ) & RanBP5 isoform-1 (residues 21 to 1116 ) \\
\hline \multicolumn{3}{|l|}{ Data collection } \\
\hline X-ray source & ESRF (ID29) & ESRF (ID29) \\
\hline Wavelength $(\AA)$ & 0.99987 & 1.00000 \\
\hline Space group & $P 2_{1} 2_{1} 2_{1}$ & $P 2_{1} 2_{1} 2_{1}$ \\
\hline$a, b, c(\AA)$ & $97.1,126.2,149.4$ & $87.1,96.3,157.0$ \\
\hline$\alpha, \beta, \gamma\left(^{\circ}\right)$ & $90,90,90$ & $90,90,90$ \\
\hline Resolution range $(\AA ̊)$ & $48-2.83(3.06-2.83)$ & $48-2.27(2.54-2.27)$ \\
\hline$R_{\text {merge }}{ }^{a}(\%)$ & $7.40(112.4)$ & $8.9(89.2)$ \\
\hline$R_{\text {pim }}(\%)$ & $3.5(50.3)$ & $4.6(45.2)$ \\
\hline$\|\sigma\|^{a}$ & $11.2(1.4)$ & $9.3(1.7)$ \\
\hline \multicolumn{3}{|l|}{ Completeness ${ }^{\mathrm{a}}(\%)$} \\
\hline Spherical & $81.0(20.3)$ & $70.3(14.5)$ \\
\hline Ellipsoidal & $94.5(56.2)$ & $92.6(68.6)$ \\
\hline Total reflections & $200,948(10184)$ & $181,499(8924)$ \\
\hline Unique reflections & 35,901 (1796) & $41,396(2070)$ \\
\hline Multiplicity ${ }^{\mathrm{a}}$ & $5.6(5.7)$ & $4.4(4.3)$ \\
\hline$C C(1 / 2)^{a}$ & $0.996(0.625)$ & $0.996(0.68)$ \\
\hline \multicolumn{3}{|l|}{ Refinement } \\
\hline No. reflection & 35,901 & 41,396 \\
\hline$R_{\text {work }} / R_{\text {free }}$ & $20.6 / 24.4$ & $19.9 / 26.0$ \\
\hline No. atoms & 8534 & 8544 \\
\hline \multicolumn{3}{|l|}{ R.m.s deviations } \\
\hline Bond length $(\AA ̊)$ & 0.009 & 0.009 \\
\hline Bond angle $\left({ }^{\circ}\right)$ & 1.04 & 0.97 \\
\hline \multicolumn{3}{|c|}{ Ramachandran statistic ${ }^{\mathbf{b}}$} \\
\hline Favoured (\%) & 93.0 & 96.0 \\
\hline Allowed (\%) & 6.4 & 3.5 \\
\hline Outlier (\%) & 0.6 & 0.5 \\
\hline \multicolumn{3}{|l|}{ Molprobity scores ${ }^{c}$} \\
\hline Score & 2.56 & 2.0 \\
\hline All-atom clashscore & 7.1 & 4.1 \\
\hline PDB code & $6 X U 2$ & 6XTE \\
\hline \multicolumn{3}{|c|}{$\begin{array}{l}\text { Values in parentheses are for highest-resolution shell. } \\
\text { a As reported by autoPROC (v1.0.5) and STARANISO (v. 2.3.20). } \\
\text { b As reported by BUSTER (v 2.10.3). } \\
\text { c As reported by MolProbity. }\end{array}$} \\
\hline
\end{tabular}


(a)

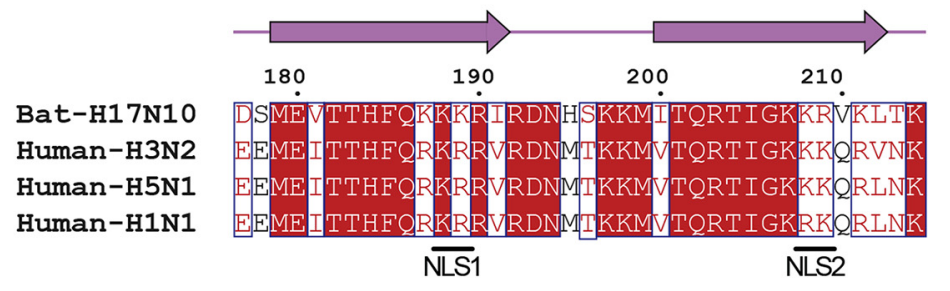

(b)

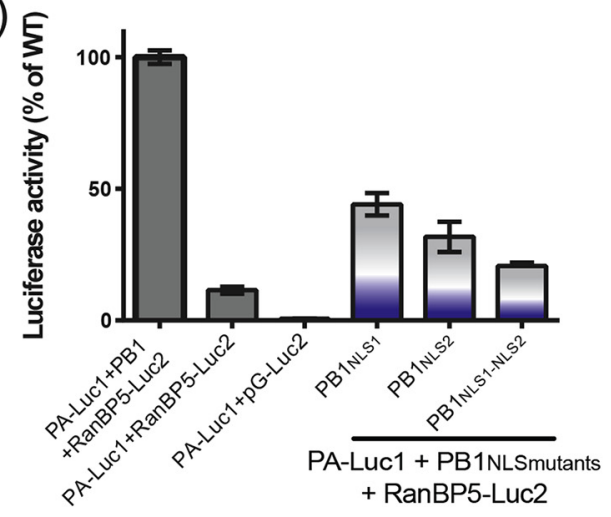

(c)

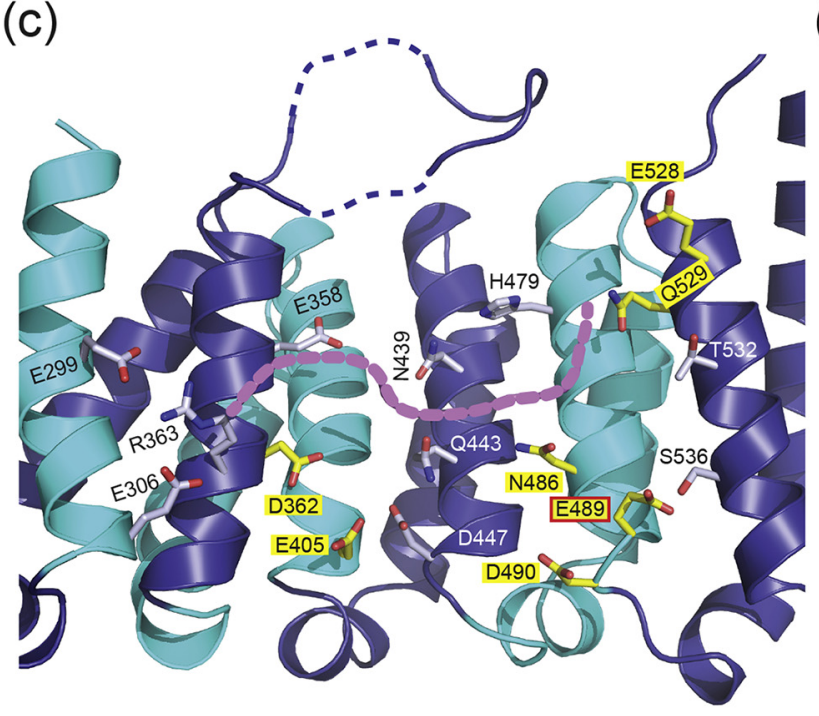

(d)

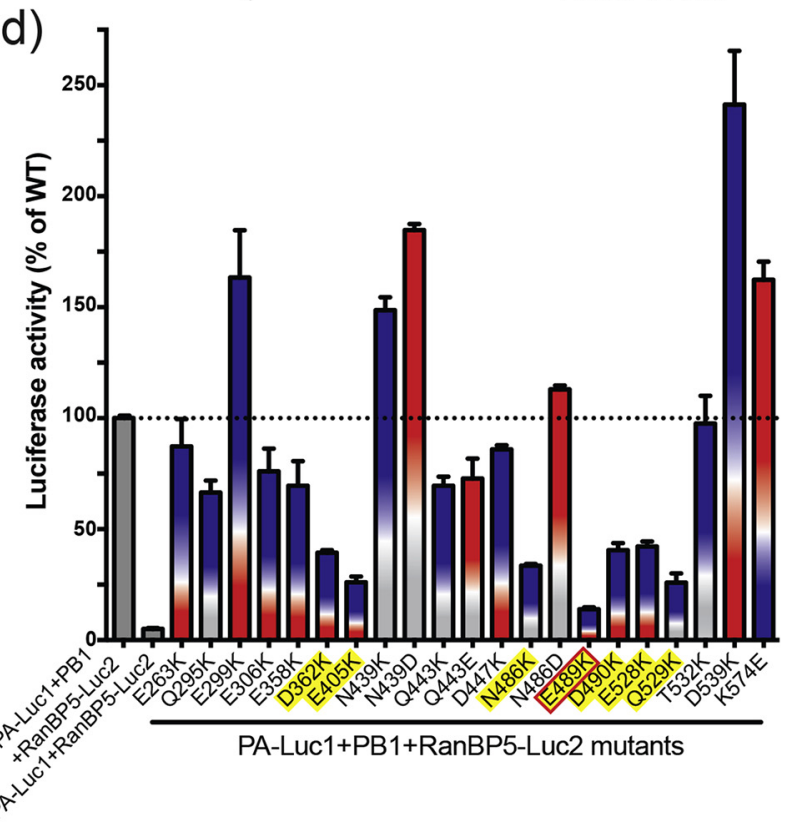

Figure 2. The cargo-binding site of RanBP5. (a) Sequence alignment of the $\beta$-sheet containing-NLS region of PB1 $\left(\mathrm{PB}_{\mathrm{NLS}}\right.$ ) from representative influenza strains (A/little yellow-shouldered bat/Guatemala/060/2010(H17N10) (BatH17N10), A/Victoria/3/1975(H3N2) (Human-H3N2), A/Viet Nam/1203/2004(H5N1) (Human-H5N1), and A/WilsonSmith/1933(H1N1) (Human-H1N1)). Secondary structure elements adopted by PB1 NLs from Bat-H17N10 (PDB code: 4WSB) is shown above the sequence alignment. Residues underlaid in red are identical between the four sequences. The position of the two basic patches (NLS1 and NLS2, respectively) identified previously as key residues for the interaction between the influenza PA-PB1 sub-complex and RanBP5 [22] are indicated below the sequence alignment. (b) Effects of $\mathrm{PB}_{\mathrm{NLS}}$ mutations on the stability of the complex between influenza PA-PB1 and RanBP5 by split G. princeps luciferasebased complementation assay. Wild-type PA fused to Gaussia Luc1 (PA-Luc1) was co-expressed with PB1 $1_{\text {NLS }}$ mutants

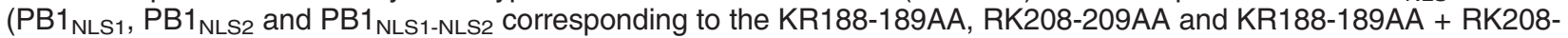
209AA mutants, respectively) and RanBP5 protein fused to Luc2 (RanBP5-Luc2) or with PB1 alone (PB1-Luc2). Substitutions of positively charged to alanyl residues are gradient-coloured from blue to grey. Luciferase activity was measured in cell lysates $24 \mathrm{~h}$ post-transfection. Data are presented as the mean luciferase activity $+/-$ the standard error of the mean of triplicates. (c) Docking of the NLS peptide of the yeast Kap121p:NLS complex structure (PDB id: 3W3X, [26]) on the ligand-free RanBP5. The NLS peptide (in pink) corresponds to a fragment of the yeast phosphate system positive regulatory protein (Pho4) docked in the N-terminal part of RanBP5 coloured alternately in cyan and blue. (d) Impact of RanBP5 mutations on the stability of the complex with influenza PA-PB1 by split G. princeps luciferase-based complementation assay. Wild-type PA fused to Luc1 (PA-Luc1) was co-expressed with PB1 and wild-type or mutant RanBP5 proteins fused to Luc2 (RanBP5-Luc2 and mutants only indicated by their amino acid substitution). pGLuc1 and pGLuc2 represent plasmids only encoding the two moieties of the Gaussia luciferase. Substitutions are gradient-coloured regarding the chemical nature of the mutations (i.e. red to blue for mutations changing negatively to positively charged residues and vice versa, grey to blue for mutations changing aliphatic to positively charged residues and grey to red for mutations changing aliphatic to negatively charged residues). Luciferase activity was measured in cell lysates $24 \mathrm{~h}$ posttransfection. Data are presented as the mean luciferase activity $+/-$ the standard error of the mean of triplicates. 
"clamp." The "clamp" acidic loop is also observed in the Kap121p structure, although the HR-18 extension leading to this loop is unstructured. Interestingly, when both isoform-3 and -1 structures are superposed, an important rearrangement of the $\mathrm{N}$-terminal region can be observed (Figure 1(b) and morphing movie, Supplementary Data), most notably in the HR-1 to HR-4. In both conformations, the HR-18 clamp forms hydrogen bonds with adjacent basic residues and locks itself in between HR-6 and HR-7 for isoform-1 and HR-7 and HR-8 for isoform-3. This conserved characteristic of bond formation suggests an important role of the HR-18 insertion in maintaining distinct conformations of RanBP5.

In order to identify the amino acids of RanBP5 that play a role in the interaction with influenza PA-PB1 sub-complex of the viral polymerase, we used the Gaussia princeps luciferase-based complementation assay (Split-Luc assay; [32,33]). We first setup and validated this assay with the influenza PA subunit fused to Luc1 and RanPB5 fused to Luc2 by introducing the mutations described by Hutchinson and co-workers [22] in the $\beta$-sheet containing NLS region of the influenza $\mathrm{PB} 1$ subunit $\left(\mathrm{PB} 1_{\mathrm{NLS}}\right.$; Figure 2(a)). Mutations in that motif strongly impair the nuclear import of the PA-PB1 sub-complex and thus viral proliferation. We observed a significant decrease in the fluorescence signal with mutations in the first and the second basic patch and a cumulative effect when mutating both patches (Figure 2(b)). Next, we took advantage of the 3D structure of the yeast Kap121p bound to an NLS and the sequence identity amongst $\beta$-importin sequences to identify a set of candidate amino acids in RanBP5 (Figure 2(c) and Figure S2). We thus quantified the interaction of PA-PB1 with about 20 RanBP5 mutants. Figure 2(d) shows that most of these point mutations drastically modulate the interaction of RanBP5 with influenza PA-PB1 sub-complex. For instance, substitutions D362K, E405K, Q443E and N486K resulted in impaired interaction, the strongest effect was obtained with E489K mutation, suggesting that these residues located in $\mathrm{HR}-8$ to $\mathrm{HR}-12$ are directly involved in PA-PB1 sub-complex recognition. We also noted that several substitutions (E229K, N439K/D, D539K and K574E) increased PA-PB1-RanBP5 interaction, possibly revealing structural flexibility and favourable conformations for binding to the PA-PB1 sub-complex. The structure of the influenza A polymerase [18] shows that the NLS on PB1 is located on a long antiparallel $\beta$-sheet (from residues 176 to 211) with the specific residues of NLS1 and NLS2 spread on the two $\beta$-strands (Figure 2(a)). Probably, this sheet should move or change when PA-PB1 binds to RanBP5. The distance between NLS1 and NLS2 is very close (20 A approximatively) to the size of NLS of Pho4 on Kap121P (Figures 2 and S2), suggesting that the NLS of PB1 (NLS1 + NLS2) probably binds as a secondary structure rather than an opened structure.
We tried to add a synthetic peptide (from residues 176 to 211) to RanBP5 (or before or after the crystallisation), but we have never able to obtain the structure of the complex. Finally, the structures of the different RdRps from influenza A-D [15-19] show that they are very flexible as is RanBP5, and we could not dock any influenza complex on the RanBP5 using our previously published SAXS curves for the complex [23].

In summary, we presented here crystallographic and mutational analyses of RanPB5, revealing important new insight in the structure, mechanism and dynamics of this karyopherin. Our crystal structures, at $3.0 \AA$ and $2.5 \AA$ resolution, respectively, provide near atomic level insight into distinct conformations adopted by isoform- 1 and -3 of this $\beta$-importin, and its remarkable flexibility, with functional implications. Our mutational analysis substantiates at the amino acid residue level the role of RanBP5 in the infectious cycle of influenza virus, delineating the molecular basis of influenza polymerase import and assembly in the nucleus. Our results thus expand our understanding of nuclear import regulating a crucial process in infectious disease. Of note, RanBP5 was shown to play a role in the infectious cycle of other viruses including HPV [34-37] and HIV [38], indicating that our observations may not be confined to influenza alone, but apply to other important human viral diseases as well.

\section{Accession codes}

Coordinates have been deposited to the PDB with IDs 6XTE (isoform-1) and 6XU2 (isoform-3).

Supplementary data to this article can be found online at https://doi.org/10.1016/j.jmb.2020.03.021.

\section{Acknowledgments}

We thank all members of our laboratories for their contributions and helpful discussions. C.S. was funded by the Hofmann-La Roche pRED external collaboration programme. This work was supported by the Agence Nationale pour la Recherche (ANR14-CE09-0017, RNAP-IAV) and the European Commission (EC) framework program 7 project ComplexINC (contract no. 279039). This study used the platforms of the Grenoble Instruct Centre (ISBG; UMS 3518 CNRS-CEA-UJF-EMBL) with support from FRISBI (ANR-10-INSB-05-02) and GRAL (ANR-10-LABX-49-01) within the Grenoble Partnership for Structural Biology.

\section{Declaration of Competing Interests}

The authors declare no conflicts of interest. 


\section{Author contributions}

Conceptualization: T.C.; Methodology: T.C., B.D. and C.B.; Investigation: C.S., B.D.C., L.S., F.G., A.A. M.C. and T.C.; Writing - Original Draft: C.S. and T.C.; Writing - Review \& Editing: T.C., C.S., B.D., C.B., I.B. and R.W.H.R.; Supervision: T.C., B.D., C.B., I.B. and R.W.H.R.; Funding Acquisition: T.C., B.D., C.B., I.B. and R.W.H.R.

Received 17 January 2020;

Received in revised form 16 March 2020;

Accepted 22 March 2020

Available online $x x x x$

Keywords:

human karyopherin;

NLS-binding site;

PA-PB1 sub-complex nuclear import; influenza polymerase assembly; host-pathogen interaction

$†$ Present address: C. Swale, Institute for Advanced Biosciences (IAB), INSERM U1209, CNRS UMR 5309, University Grenoble Alpes, 38000 Grenoble, France.

\$Present address: F. Garzoni, Imophoron Ltd., UnitDX, St. Philips Central, Albert Road, Bristol BS2 OXJ, United Kingdom.

Abbreviations used:

NPC, nuclear pore complex; NLS, nuclear localisation signal; RdRp, RNA-dependant RNA polymerase.

\section{References}

[1] D.H. Lin, A. Hoelz, The structure of the nuclear pore complex (an update), Annu. Rev. Biochem. 88 (2019) 725-783.

[2] R. Reichelt, A. Holzenburg, E.L. Buhle Jr., M. Jarnik, A. Engel, $\mathrm{U}$. Aebi, Correlation between structure and mass distribution of the nuclear pore complex and of distinct pore complex components, J. Cell Biol. 110 (1990) 883-894.

[3] I.W. Mattaj, L. Englmeier, Nucleocytoplasmic transport: the soluble phase, Annu. Rev. Biochem. 67 (1998) 265-306.

[4] D. Gorlich, U. Kutay, Transport between the cell nucleus and the cytoplasm, Annu. Rev. Cell Dev. Biol. 15 (1999) 607-660.

[5] E.P. Lei, P.A. Silver, Protein and RNA export from the nucleus, Dev. Cell 2 (2002) 261-272.

[6] L.F. Pemberton, G. Blobel, J.S. Rosenblum, Transport routes through the nuclear pore complex, Curr. Opin. Cell Biol. 10 (1998) 392-399.

[7] F. Bono, A.G. Cook, M. Grunwald, J. Ebert, E. Conti, Nuclear import mechanism of the EJC component Mago-Y14 revealed by structural studies of importin 13, Mol. Cell 37 (2010) 211-222.

[8] A. Padavannil, P. Sarkar, S.J. Kim, T. Cagatay, J. Jiou, C. A. Brautigam, et al., Importin-9 wraps around the H2A-H2B core to act as nuclear importer and histone chaperone, Elife. 8 (2019).

[9] M. Floer, G. Blobel, M. Rexach, Disassembly of RanGTPkaryopherin beta complex, an intermediate in nuclear protein import, J. Biol. Chem. 272 (1997) 19538-19546.
[10] F. Melchior, B. Paschal, J. Evans, L. Gerace, Inhibition of nuclear protein import by nonhydrolyzable analogues of GTP and identification of the small GTPase ran/TC4 as an essential transport factor, J. Cell Biol. 123 (1993) 1649-1659.

[11] M.S. Moore, G. Blobel, The GTP-binding protein Ran/TC4 is required for protein import into the nucleus, Nature. 365 (1993) 661-663.

[12] S. Boulo, H. Akarsu, R.W. Ruigrok, F. Baudin, Nuclear traffic of influenza virus proteins and ribonucleoprotein complexes, Virus Res. 124 (2007) 12-21.

[13] J.F. Cros, P. Palese, Trafficking of viral genomic RNA into and out of the nucleus: influenza, Thogoto and Borna disease viruses, Virus Res. 95 (2003) 3-12.

[14] I.M. Jones, P.A. Reay, K.L. Philpott, Nuclear location of all three influenza polymerase proteins and a nuclear signal in polymerase PB2, EMBO J. 5 (1986) 2371-2376.

[15] H. Fan, A.P. Walker, L. Carrique, J.R. Keown, I. Serna Martin, D. Karia, et al., Structures of influenza A virus RNA polymerase offer insight into viral genome replication, Nature. 573 (2019) 287-290.

[16] N. Hengrung, K. El Omari, I. Serna Martin, F.T. Vreede, S. Cusack, R.P. Rambo, et al., Crystal structure of the RNAdependent RNA polymerase from influenza C virus, Nature. 527 (2015) 114-117.

[17] Q. Peng, Y. Liu, R. Peng, M. Wang, W. Yang, H. Song, et al., Structural insight into RNA synthesis by influenza D polymerase, Nat. Microbiol. 4 (2019) 1750-1759.

[18] A. Pflug, D. Guilligay, S. Reich, S. Cusack, Structure of influenza A polymerase bound to the viral RNA promoter, Nature. 516 (2014) 355-360.

[19] S. Reich, D. Guilligay, A. Pflug, H. Malet, I. Berger, T. Crépin, et al., Structural insight into cap-snatching and RNA synthesis by influenza polymerase, Nature. 516 (2014) 361-366.

[20] T. Deng, J. Sharps, E. Fodor, G.G. Brownlee, In vitro assembly of PB2 with a PB1-PA dimer supports a new model of assembly of influenza $A$ virus polymerase subunits into a functional trimeric complex, J. Virol. 79 (2005) 8669-8674.

[21] T. Deng, O.G. Engelhardt, B. Thomas, A.V. Akoulitchev, G. G. Brownlee, E. Fodor, Role of ran binding protein 5 in nuclear import and assembly of the influenza virus RNA polymerase complex, J. Virol. 80 (2006) 11911-11919.

[22] E.C. Hutchinson, O.E. Orr, S. Man Liu, O.G. Engelhardt, E. Fodor, Characterization of the interaction between the influenza A virus polymerase subunit PB1 and the host nuclear import factor Ran-binding protein 5, J Gen Virol. 92 (2011) 1859-1869.

[23] C. Swale, A. Monod, L. Tengo, A. Labaronne, F. Garzoni, J. M. Bourhis, et al., Structural characterization of recombinant IAV polymerase reveals a stable complex between viral PA-PB1 heterodimer and host RanBP5, Sci. Rep. 6 (2016) 24727.

[24] F. Tarendeau, J. Boudet, D. Guilligay, P. Mas, C. Bougault, S. Boulo, et al., Structure and nuclear import function of the C-terminal domain of influenza virus polymerase PB2 subunit, Nat. Struct. Mol. Biol. 14 (2007) 229-233.

[25] J. Kobayashi, H. Hirano, Y. Matsuura, Crystal structure of the karyopherin Kap121p bound to the extreme C-terminus of the protein phosphatase Cdc14p, Biochem. Biophys. Res. Commun. 463 (2015) 309-314.

[26] J. Kobayashi, Y. Matsuura, Structural basis for cell-cycledependent nuclear import mediated by the karyopherin Kap121p, J. Mol. Biol. 425 (2013) 1852-1868.

[27] A.J. McCoy, R.W. Grosse-Kunstleve, P.D. Adams, M.D. Winn, L.C. Storoni, R.J. Read, Phaser crystallographic software, J. Appl. Crystallogr. 40 (2007) 658-674. 
[28] S. An, J. Yoon, H. Kim, J.J. Song, U.S. Cho, Structure-based nuclear import mechanism of histones $\mathrm{H} 3$ and $\mathrm{H} 4$ mediated by Kap123, Elife. 6 (2017).

[29] G. Cingolani, C. Petosa, K. Weis, C.W. Muller, Structure of importin-beta bound to the IBB domain of importin-alpha, Nature. 399 (1999) 221-229.

[30] T. Imasaki, T. Shimizu, H. Hashimoto, Y. Hidaka, S. Kose, N. Imamoto, et al., Structural basis for substrate recognition and dissociation by human transportin 1, Mol. Cell 28 (2007) 57-67.

[31] M. Koyama, N. Shirai, Y. Matsuura, Structural insights into how Yrb2p accelerates the assembly of the Xpo1p nuclear export complex, Cell Rep. 9 (2014) 983-995.

[32] P. Cassonnet, C. Rolloy, G. Neveu, P.O. Vidalain, T. Chantier, J. Pellet, et al., Benchmarking a luciferase complementation assay for detecting protein complexes, Nat. Methods 8 (2011) 990-992.

[33] G. Fournier, C. Chiang, S. Munier, A. Tomoiu, C. Demeret, P.O. Vidalain, et al., Recruitment of RED-SMU1 complex by influenza $A$ virus RNA polymerase to control viral mRNA splicing, PLoS Pathog. 10 (2014), e1004164.
[34] L.M. Nelson, R.C. Rose, J. Moroianu, The L1 major capsid protein of human papillomavirus type 11 interacts with Kap beta2 and Kap beta3 nuclear import receptors, Virology. 306 (2003) 162-169.

[35] M.S. Darshan, J. Lucchi, E. Harding, J. Moroianu, The 12 minor capsid protein of human papillomavirus type 16 interacts with a network of nuclear import receptors, J. Virol. 78 (2004) 12179-12188.

[36] K. Klucevsek, J. Daley, M.S. Darshan, J. Bordeaux, J. Moroianu, Nuclear import strategies of high-risk HPV18 L2 minor capsid protein, Virology. 352 (2006) 200-208.

[37] E. Krawczyk, J.A. Hanover, R. Schlegel, F.A. Suprynowicz, Karyopherin beta3: a new cellular target for the HPV-16 E5 oncoprotein, Biochem. Biophys. Res. Commun. 371 (2008) 684-688.

[38] M. Arnold, A. Nath, J. Hauber, R.H. Kehlenbach, Multiple importins function as nuclear transport receptors for the Rev protein of human immunodeficiency virus type 1, J. Biol. Chem. 281 (2006) 20883-20890. 